\title{
Four spices prevent mice from contracting Salmonella enterica serovar Typhimurium
}

\author{
CHIUNG-HUNG CHANG ${ }^{1,2}$, JIA-HUA FU ${ }^{3}$, CHIU-HSIAN SU ${ }^{4}$, MEI-CHIN YIN ${ }^{5 *}$ and YUAN-MAN HSU ${ }^{4 *}$ \\ ${ }^{1}$ Department of Traditional Chinese Medicine, Tainan Municipal Hospital (Managed by Show Chwan Medical Care Corporation), \\ Tainan 70173; ${ }^{2}$ Department of Traditional Chinese Medicine, Taichung Veterans General Hospital, Taichung 40705; \\ Departments of ${ }^{3}$ Nutrition and ${ }^{4}$ Biological Science and Technology, China Medical University, Taichung 40402; \\ ${ }^{5}$ Department of Food Nutrition and Health Biotechnology, Asia University, Taichung 41354, Taiwan, R.O.C.
}

Received October 9, 2018; Accepted May 16, 2019

DOI: $10.3892 /$ etm.2019.7892

\begin{abstract}
Ligustrum lucidum Ait (LL), Lysimachia christinae Hance (LC), Mentha piperita Linn (MP), and Cinnamomum cassia Presl (CC) are common spices used in Asia. The present study investigated the anti-Salmonella effects of the four spices using aqueous extracts. The amount of phenolic acids and flavonoids in each spice aqueous extract was determined as indicators of purity. Mice were pretreated with LL, LC, MP or CC aqueous extract for 7 days. Following infection with Salmonella enterica serovar Typhimurium strain ST21 (ST21), the aqueous extract of each spice was subsequently administered for 4 days. ST21 infected mice had lower body weight compared with the control group. The administration of spice aqueous extracts significantly increased body weight following infection. ST21 infection increased the fecal ST21 counts compared with the control group; however, following spice aqueous extract treatments, ST21 counts significantly decreased. The spice treatments also significantly reduced ST21 count in blood and the organs. Notably, ST21 infection increased interferon (IFN) $-\gamma$ and interleukin (IL)- 6 levels in serum whilst spice treatments reduced these cytokines. In the spleen, spice treatment significantly lowered IFN- $\gamma$, IL-6, IL-1 $\beta$, and tumor necrosis factor (TNF)- $\alpha$ levels, but increased IL-12 levels. ST21 infection stimulated the production of immunoglobulin ( $\mathrm{Ig}) \mathrm{A}$ and IgM in serum whilst spice aqueous extract treatment significantly decreased these levels. In summary, LL and MP aqueous extract treatments had the most significant effect in protecting against ST21 infection. Results of the RAW 264.7 cell infection
\end{abstract}

Correspondence to: Dr Yuan-Man Hsu, Department of Biological Science and Technology, China Medical University, 91 Hsueh-Shih Road, Taichung 40402, Taiwan, R.O.C.

E-mail: yuanmh@mail.cmu.edu.tw

${ }^{*}$ Contributed equally

Key words: Salmonella enterica serovar Typhimurium, Ligustrum lucidum Ait, Mentha piperita Linn, Cinnamomum cassia Presl, bactericidal activity, virulence attenuation model suggested that the mechanisms involved in the anti-ST21 effect of each spice were individually different. All four aqueous extracts demonstrated different mechanisms in attenuating ST21 invasion with the protective effect of LC aqueous extract potentially involving TNF- $\alpha$ expression. The present findings suggested that the four spices may be considered as potent functional foods due to their anti-Salmonella effects.

\section{Introduction}

Salmonella, belongs to Gram-negative bacteria and can cause enteric diseases in humans and animals including pigs and chickens. Salmonella enterica serovar Typhimurium (ST) induces severe acute gastroenteritis, diarrhea, and fever in humans and is often caused by consumption of contaminated food or water (1). Therefore, the identification and development of agents with anti-ST effects is of great importance.

ST invades mammalian cells such as macrophages and epithelial cells, and the bacteria subsequently adopt strategies that enable their intracellular survival within the host cells. This typically involves the identification and resistance of the host innate immune system, which is essential for ST-induced pathogenesis (2). ST infection can induce a range of host immune responses for example lymphocyte proliferation and antibody generation $(3,4)$. The release of cytokines including interferon (IFN)- $\gamma$, tumor necrosis factor (TNF)- $\alpha$, and interleukin (IL)-6 leads to inflammation (5). The levels of inflammatory cytokines and immunoglobulin (Ig)s in ST-infected hosts are regarded as a good gauge of infection status. Conversely, a decrease in inflammatory cytokines and Igs in circulation and in the host organs are useful markers for evaluating potential activity of anti-ST agents.

Ligustrum lucidum Ait (LL), Lysimachia christinae Hance (LC), Mentha piperita Linn (MP), and Cinnamomum cassia Presl (CC) are four edible plant foods commonly used for cooking or folk medicine in many East Asian countries including China, Japan and Taiwan. It is reported that these spices contain phytochemicals such as phenolic acids and flavonoids $(6,7)$ that display antioxidant and anti-inflammatory activities (8). Furthermore, LL exerts an anti-inflammatory effect via the inhibition of TNF- $\alpha$ production in mouse peritoneal macrophages (9). In addition, MP aqueous extract arrests the growth 
of bacteria such as Staphylococcus aureus, Pseudomonas aeruginosa, and Bordetella bronchiseptica (10). The inhibitory activities of MP and CC essential oil extracts on ST have been previously investigated $(11,12)$; however, the application of aqueous extracts against ST infection is more appropriate as the natural properties remain unaltered.

Using aqueous extracts of common spices against food-borne diseases should be more practical for daily life in comparison to extracts prepared via chemical purification processes. Currently, there are few reports regarding the protective effects of LL, LC, MP and CC aqueous extracts against ST infection. In order to further understand and evaluate the application of these spices, the total content of phenolic acids and flavonoids in these aqueous extracts was taken as indicators of purity. An in vivo and in vitro study was conducted to investigate the anti-ST effects of LL, LC, MP and CC aqueous extracts.

\section{Materials and methods}

Materials. S. enterica serovar Typhimurium strain ST21 (ST21) was kindly supplied by Dr. Chao-chin Chang from National Chung Hsing University (13). Bacteria were grown in Luria Bertani (LB) broth to log stationary phase $(15,000 \mathrm{x}$ g shaking; $37^{\circ} \mathrm{C}$ ) for $8 \mathrm{~h}$ to $\mathrm{OD}_{600 \mathrm{~nm}}$ of 0.8 . Following harvesting by centrifugation at $8,000 \mathrm{xg}$, the bacterial pellet was resuspended in PBS, and adjusted to a final concentration of $10^{10}$ colony forming units (CFU)/ml in PBS. Broth and agar were obtained from Difco Laboratories Inc. Dry LL, LC, MP, and CC were purchased from Ka-da Spice Store. Aqueous extracts were obtained from the dried ripe fruits of LL, dried whole plants of LC, dried leaves of MP, and dried barks of CC (Fig. 1). A total of $10 \mathrm{~g}$ of each spice were chopped and mixed thoroughly with $400 \mathrm{ml} d \mathrm{H}_{2} \mathrm{O}$ in a waring blender at room temperature for $5 \mathrm{~min}$. The mixture was then boiled in $d \mathrm{H}_{2} \mathrm{O}$ for $30 \mathrm{~min}$. Following cooling down to the room temperature, the aqueous extract was collected by filtration through a Whatman No. 1 filter paper, freeze-dried to a fine powder finally resuspended in $10 \mathrm{ml} d \mathrm{H}_{2} \mathrm{O}$.

Determination of total phenolic acid and flavonoid contents. The method described in Sreelatha and Padma (14) was used to determine the total phenolic acid and flavonoid content in aqueous extract powder. Results were expressed as gallic acid and quercetin equivalent, respectively. This assay was repeated in triplicate.

Anti-microbial activity. The minimum inhibitory concentrations and minimum bactericidal concentrations of spice aqueous extracts were tested by a two-fold serial dilution method (15). Aqueous extracts were serially diluted with PBS to achieve concentrations of $12.5,25,50,100$ and $200 \mathrm{mg} / \mathrm{ml}$. ST21 was cultured in Mueller Hinton Broth then diluted to $10^{6} \mathrm{CFU} / \mathrm{ml}$ in PBS determined by absorbance at $\mathrm{OD}_{600 \mathrm{~nm}}$. Equal volumes of ST21 suspension and diluted spice aqueous extract $(100 \mu \mathrm{l})$ were mixed and added to a 96-well plate, with an additional well containing only broth used as a negative control. The plate was incubated at $37^{\circ} \mathrm{C}$ for $24 \mathrm{~h}$, then the well with the lowest concentration of spice aqueous extract that demonstrated no visible bacterial growth was considered as the MIC. All spice aqueous extract samples that demonstrated complete inhibition of visual bacterial growth were identified then $10 \mu \mathrm{l}$ of each culture was transferred onto a LB agar plate and incubated for $16 \mathrm{~h}$ at $37^{\circ} \mathrm{C}$. The complete visual absence of bacterial colonies on the agar surface of samples at the lowest spice aqueous extract concentration was defined as the MBC. Each assay was repeated in triplicate.

Animal experiment. A total of 60 male 8-week-old Balb/c mice weighing 20-22 g were purchased from National Laboratory Animal Center. Mice were housed in 12-h light/dark cycles with access to food and water ad libitum. The study was approved by the China Medical University animal care and use committee (permission no. 105-23).

Mice were randomly divided into six groups $(n=10)$ : The infection and control group mice received the normal diet with and without ST21 infection, respectively; the treatment group mice, including LL, LC, MP, and CC groups, received different spice treatments with ST21 infection. A total of $100 \mu 1$ freshly prepared aqueous extract at concentration of $50 \mathrm{mg} / \mathrm{ml}$ was applied orally to mice by the feeding tube for 7 days, once a day. At day 7, following a 12-h fast, each mouse was infected by oral injection of $200 \mu 1 \mathrm{PBS}$ containing ST21 at $5 \times 10^{10} \mathrm{CFU}$. Following infection, aqueous extract of each spice (200 $\mu \mathrm{l})$ was continuously supplied for 4 days. Therefore, there was a 7-day pre-treatment with spice aqueous extracts before the infection, and a 4-day post-treatment following infection. Feces were collected on day 8-11. Body weight was recorded one day before the experiment (day 0), the day of infection (day 7), and the last day of treatment (day 11). Following the 4-day treatment, mice were fasted overnight and sacrificed in the morning. Blood $(1 \mathrm{ml})$ was obtained from the heart via microsyringe and the serum was immediately separated. The liver, spleen and small intestine from each mouse were collected. Each organ tissue (100 mg) was mixed with $2 \mathrm{ml}$ of PBS and then homogenized by a tissue homogenizer (Glas-Col Co.). The homogenate was collected via filtration through a Whatman No. 1 filter paper in preparation for further experiments.

ST21 count in feces, blood and organs. Following ST21 infection, fecal samples were collected daily from day 8-11 prior to treatment. The fecal matter was processed within $2 \mathrm{~h}$ of sampling. ST21 per gram of feces was determined. In brief, fecal samples $(1 \mathrm{~g})$ were collected in $10 \mathrm{ml}$ PBS. Fecal suspension, blood and organ homogenate at $100 \mu 1$ was serially diluted in PBS, then plated on Salmonella-Shigella (SS) agar plates. Following incubation overnight at $37^{\circ} \mathrm{C}, \mathrm{CFU}$ were counted.

Measurement of inflammatory factor levels. IFN- $\gamma$ and IL-6 serum levels, and IFN- $\gamma$, IL-6, IL- $1 \beta$, TNF- $\alpha$, and IL-12 levels in spleen homogenate of all mice and culture supernatant collected from the $H$. pylori-infected RAW264.7 cells were measured. These cytokines were analyzed using mouse IFN- $\gamma$ ELISA kit (cat. no. 88-7314-88), mouse IL-6 ELISA kit (cat. no. 88-7064-88), mouse IL-1 $\beta$ ELISA kit (cat. no. 88-7013-88), mouse TNF- $\alpha$ ELISA kit (cat. no. 88-7324-88) and mouse IL-12 ELISA kit (cat. no. 88-7121-88; all Invitrogen; Thermo Fisher Scientific, Inc.). Samples were run in duplicates according to manufacturer's protocol. 
(A)

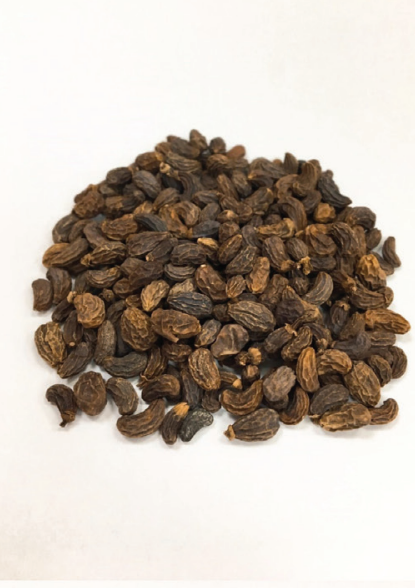

(C)

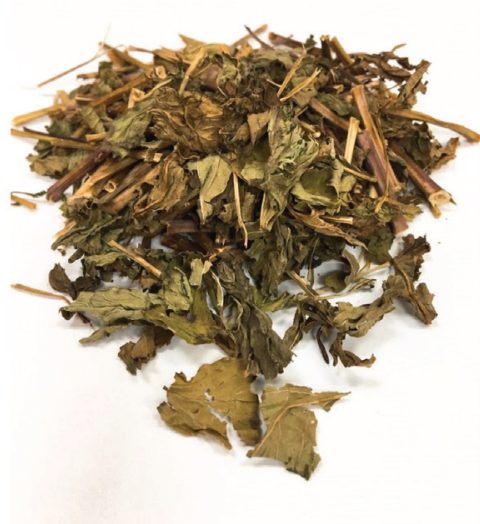

(B)

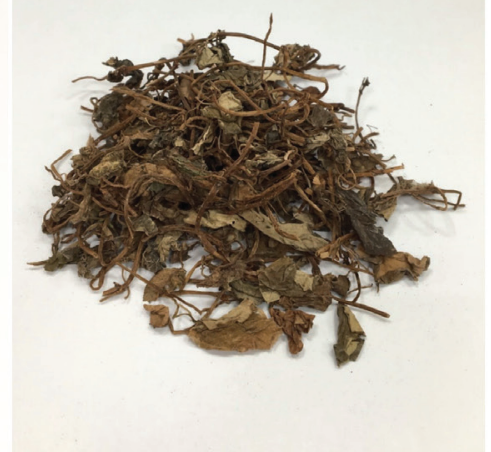

(D)

Figure 1. Aqueous extracts were prepared from (A) dried ripe fruits of Ligustrum lucidum Ait, (B) dried whole plants of Lysimachia christinae Hance, (C) dried aerial part of Mentha piperita Linn, and (D) dried bark of Cinnamomum cassia Presl.

ST21 specific Ig detection. Anti-ST21-specific IgA and IgM in the sera of treated mice were detected using ELISA according to the method of Chang et al (13), which was briefly described as follows. The 96-well plates were coated with $100 \mu \mathrm{l} /$ well of heat-killed ST21 harvested from a bacterial culture $\left(1 \times 10^{11} \mathrm{CFU} / \mathrm{ml}\right)$ at 1:100 dilution in carbonate/bicarbonate coating buffer ( $\mathrm{pH} 9.6$ ) at $4^{\circ} \mathrm{C}$ overnight. Coated plates were first blocked with BSA buffer (1\% of BSA in PBS) at room temperature for $2 \mathrm{~h}$, then washed 5 times in PBS containing $0.1 \%$ Tween 20 (PBST). A total of $100 \mu \mathrm{l} /$ well of mouse serum collected 4 days post-treatment were diluted in BSA buffer (1:10) was then added to the coated plates for $2 \mathrm{~h}$ at room temperature. Following five washes with PBST, $100 \mu \mathrm{l} /$ well of peroxidase-conjugated goat anti-mouse $\operatorname{Ig}$ A (cat. no. A90-103P) or IgM (cat. no. A90-101P) antibodies (diluted 1:5,000; Bethyl Laboratories, Inc.) were added to each well and plates were incubated at room temperature for $1 \mathrm{~h}$. Following five washes, $100 \mu \mathrm{l} /$ well of tetramethylbenzidine $(1 \mathrm{mg} / \mathrm{ml}$ in DMSO; KPL, Inc.) was added and incubated for $30 \mathrm{~min}$ at room temperature. Then $100 \mu \mathrm{l} /$ well of stop solution $\left(2 \mathrm{M} \mathrm{N}_{2} \mathrm{SO}_{4}\right)$ was added and the $\mathrm{OD}$ at $450 \mathrm{~nm}$ was measured. Each assay in this experiment was repeated in triplicate.

Invasion of $H$. pylori into RAW264.7 cells. The murine monocyte/macrophage cell line RAW264.7 (cat. no. TIB-71) was obtained from American Type Culture Collection. RAW264.7 cells were seeded into 96-well plates at a density of $1 \times 10^{5}$ cells/well then cultured at $5 \% \mathrm{CO}_{2}$ in Dulbecco's modified Eagle's medium (DMEM; cat. no. 12100-046; Gibco; Thermo Fisher Scientific, Inc.) supplemented with $10 \%$ fetal bovine serum for $18 \mathrm{~h}$ at $37^{\circ} \mathrm{C}$. The culture supernatant was removed then $100 \mu \mathrm{l}$ of antibiotic-free DMEM was added to each well. RAW264.7 cells were then infected with ST21 at a multiplicity of infection (MOI) of 10. For the co-incubation group, the cells were treated with spice aqueous extracts at a final concentration of $5 \mathrm{mg} / \mathrm{ml}$ alongside ST21 infection for $1 \mathrm{~h}$ at $37^{\circ} \mathrm{C}$. For the pretreatment groups, prior to infection, RAW264.7 cells (cell-pretreatment group) or ST21 (ST21-pretreatment group) were pretreated with LL, LC, MP and CC aqueous extract at final concentration of $5 \mathrm{mg} / \mathrm{ml}$ for $1 \mathrm{~h}$ at $37^{\circ} \mathrm{C}$; infection was performed as described. For TNF- $\alpha$ depletion, anti-mouse TNF- $\alpha$ capture antibodies (1:100; cat. no. 14-7423-68A; Invitrogen; Thermo Fisher Scientific, Inc.) were added in the cell culture treated with spice aqueous extracts for $1 \mathrm{~h}$ at $37^{\circ} \mathrm{C}$; the same procedure was performed in the co-incubation group. Cell-associated bacteria were then quantified $1 \mathrm{~h}$ following infection. Briefly, to determine the number of viable intracellular bacteria, infected cells was washed three times in PBS then incubated with $100 \mu \mathrm{g} / \mathrm{ml}$ of the membrane-impermeable antibiotic gentamicin (Sigma-Aldrich; Merck KGaA) for $1.5 \mathrm{~h}$ at 
Table I. Content (mg/g of dry spices) of total phenolic acids and total flavonoids in aqueous extract of LL, LC, MP or CC.

\begin{tabular}{lcc}
\hline Spice & Total phenolic acids & Total flavonoids \\
\hline LL & $1.05 \pm 0.12$ & $2.91 \pm 0.34$ \\
LC & $1.37 \pm 0.21$ & $3.16 \pm 0.41$ \\
MP & $1.58 \pm 0.21$ & $7.65 \pm 0.86$ \\
CC & $0.50 \pm 0.09$ & $1.67 \pm 0.31$ \\
\hline
\end{tabular}

The data are expressed as mean \pm standard deviation $(n=3)$. LL, Ligustrum lucidum Ait; LC, Lysimachia christinae Hance; MP, Mentha piperita Linn; CC, Cinnamomum cassia Presl.

$37^{\circ} \mathrm{C}$ to remove extracellular bacteria. Cell culture supernatants were removed following centrifugation ( $5 \mathrm{~min}$; 1,500 x g; room temperature). Cells were washed with PBS twice, and osmotic lysis was performed to calculate the total quantity of bacteria remaining. In brief, $d \mathrm{H}_{2} \mathrm{O}$ was added to the infected cells following washing, the cell lysates were re-suspended in PBS then plated using serial dilutions on the SS agar plates (Difco; BD Biosciences). These plates were cultured with $100 \mu \mathrm{l}$ from each dilution at $37^{\circ} \mathrm{C}$ for $18 \mathrm{~h}$. Bacterial cell numbers were then determined by manual colony counting. Results were expressed as a percentage of the invasion activity of ST21 in comparison with the infection group. The supernatants of ST21-infected RAW264.7 cells treated with spices were analyzed for cytokines. The concentrations shown in Table I are also the lowest concentrations for different batches of spices eligible for the current study.

Statistical analysis. Data were analyzed using SPSS v12.0 software (SPSS, Inc.) Statistical significance was assessed between two groups using Student's t-test or between multiple group using one-way analysis of variance followed by Dunnett's post hoc test. Results were presented as the mean \pm standard deviation. $\mathrm{P}<0.05$ was considered to indicate statistical significance.

\section{Results}

Total phenolic acid and flavonoid content. In order to confirm the purity of aqueous extracts that was prepared, the total phenolic acid and flavonoid content in spice aqueous extracts was determined. Total phenolic acid content in LL, LC, MP and $\mathrm{CC}$ aqueous extracts was in the range of $0.50-1.58 \mathrm{mg} / \mathrm{g}$ of spice (Table I). Total flavonoid content in LL, LC, MP and $\mathrm{CC}$ aqueous extract was in the range of $1.67-7.65 \mathrm{mg} / \mathrm{g}$ of spice (Table I). MP contained the highest amount of total phenolic acids and flavonoids whilst $\mathrm{CC}$ had the lowest amount.

Spice aqueous extracts exhibit bactericidal effects. Anti-ST21 activity was examined to determine the bactericidal activities of LL, LC, MP and CC aqueous extracts. MIC were in the range of $125-500 \mathrm{mg} / \mathrm{ml}$ and $\mathrm{MBC}$ values were $>500 \mathrm{mg} / \mathrm{ml}$ (Table II). LL and MP aqueous extracts exhibited a stronger inhibitory effect on the growth of ST21. However, it is important to note that no spice aqueous extract was able to eradicate ST21 at concentration of $<500 \mathrm{mg} / \mathrm{ml}$.
Table II. MICs and MBCs of LL, LC, MP, or CC against ST21.

\begin{tabular}{lcc}
\hline Spice & MIC $(\mathrm{mg} / \mathrm{ml})$ & MBC $(\mathrm{mg} / \mathrm{ml})$ \\
\hline LL & 125 & $>500$ \\
LC & 500 & $>500$ \\
MP & 125 & $>500$ \\
CC & 500 & $>500$ \\
\hline
\end{tabular}

MIC, minimum inhibitory concentrations; MBC, minimum bactericidal concentrations; LL, Ligustrum lucidum Ait; LC, Lysimachia christinae Hance; MP, Mentha piperita Linn; CC, Cinnamomum cassia Pres1; ST21, Salmonella enterica serovar Typhimurium strain ST21.

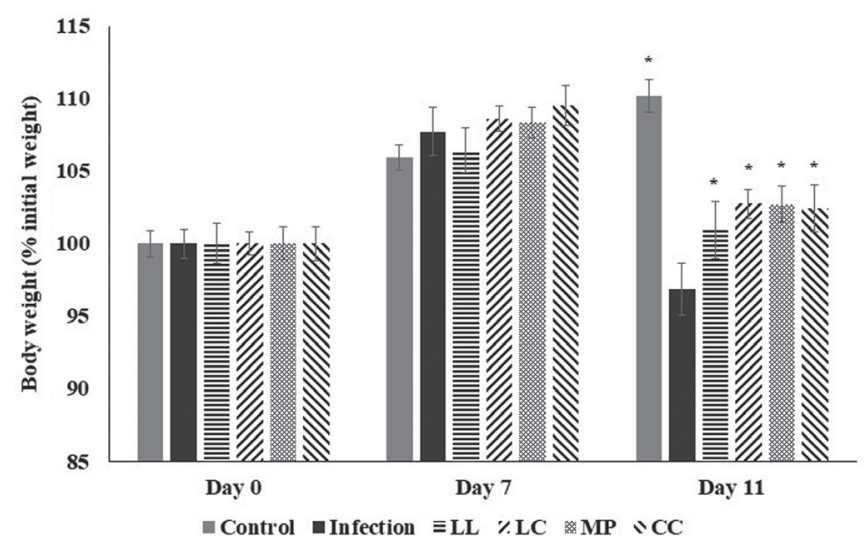

Figure 2. Body weight of mice following infection with ST21 then treatment with LL, LC, MP, and CC aqueous extract. Body weight of mice without infection (control), ST21-infected mice without any treatment (infection), or following LL, LC, MP or CC aqueous extract treatment ( $\mathrm{n}=10)$. ${ }^{*} \mathrm{P}<0.05$ vs. the infection group on the same day. ST21, Salmonella enterica serovar Typhimurium strain ST21; LL, Ligustrum lucidum Ait; LC, Lysimachia christinae Hance; MP, Mentha piperita Linn; CC, Cinnamomum cassia Presl.

Spice aqueous extracts maintain body weight following infection. ST infection in mice causes murine typhoid that results in a reduction of body weight $(1,16)$. In the present study, four days following ST21 infection (day 11), infected mice had lower body weight compared with the control group (Fig. 2; $\mathrm{P}<0.05)$. However, treatment with LL, LC, MP and CC aqueous extracts significantly increased body weight compared with the infection group (Fig. 2; $\mathrm{P}<0.05$ ). There was no significant difference in body weight between any groups in days 0 and 7 (Fig. 2; $\mathrm{P}>0.05)$.

Spice aqueous extracts decrease ST21 count infeces, blood, and organs. Following oral infection of mice with ST, bacteria replicate quickly within the guts then are subsequently eliminated in the feces (2). ST can also spread from the guts to the liver, spleen and blood circulatory system $(1,16)$. It has been reported that the ST viable count reaches the maximum amount four days following oral inoculation (17). In the present study the ST21 count in feces at 1-4 days following infection (day 8-11) was increased when compared with control group $(\mathrm{P}<0.05$; Fig. 3A). Between days 8-11, fecal ST21 counts in groups that received LL, LC, MP and CC treatment were lower compared 
A
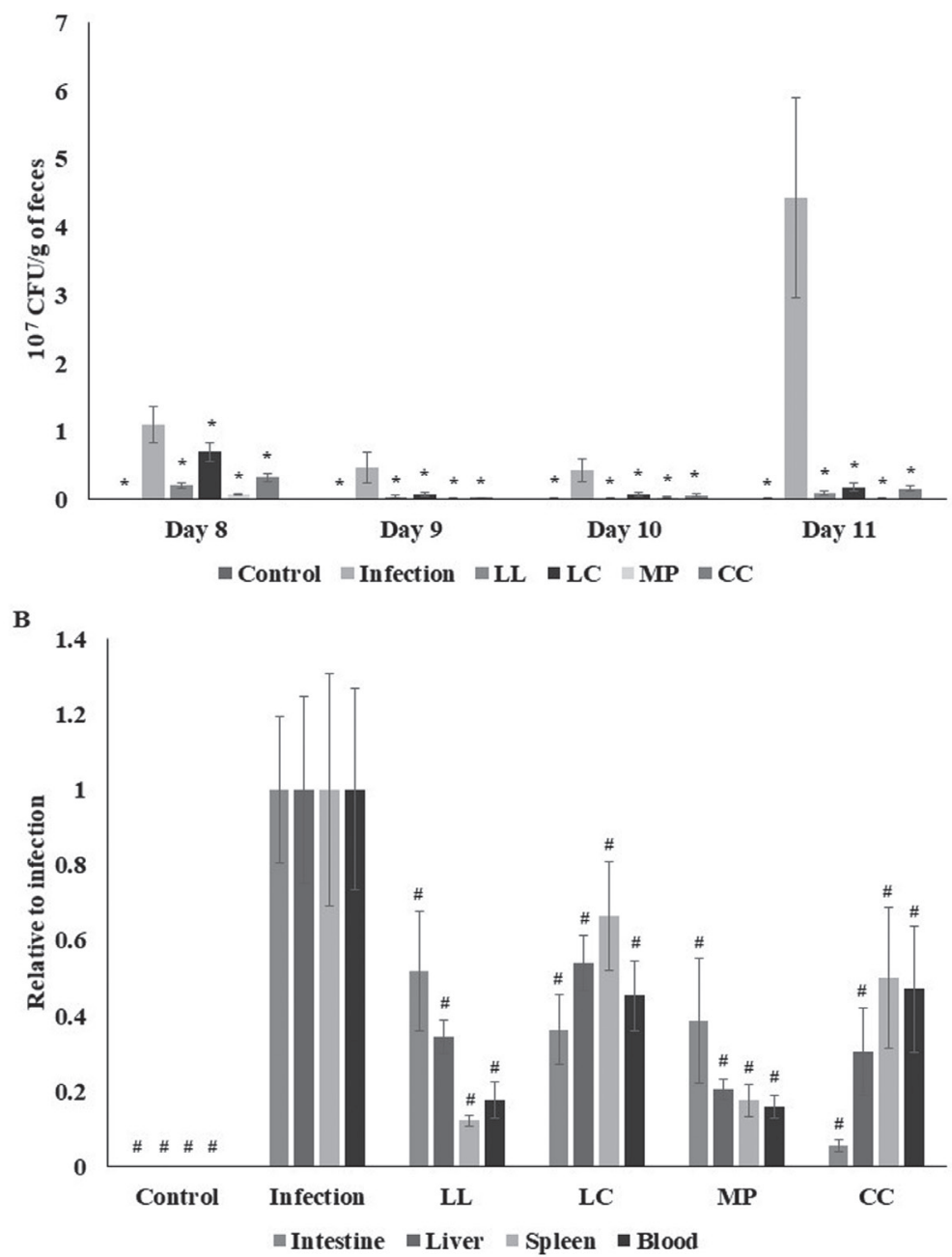

Figure 3. Spice aqueous extracts decrease ST21 count in feces, blood, and organs. (A) ST21 counts in feces, (B) blood, small intestine, liver and spleen of mice without infection (control), ST21-infected mice without any treatment (infection), or following LL, LC, MP or CC aqueous extract treatment (n=10). ${ }^{*} \mathrm{P}<0.05$ vs. the infection group on the same day. ${ }^{\text {"P }}<0.05$ vs. the infection group in the same organ. ST21, Salmonella enterica serovar Typhimurium strain ST21; LL, Ligustrum lucidum Ait; LC, Lysimachia christinae Hance; MP, Mentha piperita Linn; CC, Cinnamomum cassia Presl; CFU, colony forming units.

with the infection group ( $\mathrm{P}<0.05$; Fig. 3A). ST21 counts in the small intestine, liver, spleen and blood significantly decreased following all spice treatments $(\mathrm{P}<0.05$; Fig. 3B).

Spice aqueous extracts attenuate inflammatory stress and decrease ST21 specific Igs. ST clearance involves inflammation and Ig associated reactions therefore, monitoring inflammatory cytokines and Igs in circulation and in host organs are necessary in evaluating potential anti-ST activity. ST21 infection significantly increased IFN- $\gamma$ and IL-6 levels in the serum $(\mathrm{P}<0.05$; Fig. 4A) whilst LL, LC, MP and CC aqueous extract treatments significantly reduced the serum IFN- $\gamma$ and IL- 6 levels compared with the infection group $(\mathrm{P}<0.05$; Fig. 4A). In the spleen, the spice aqueous extract treatments significantly decreased IFN- $\gamma$, IL-6, IL- $1 \beta$, and TNF- $\alpha$ levels; however, there was an increase in IL-12 level (Fig. 4B; $\mathrm{P}<0.05)$ whilst LC treatment did not suppress the IFN- $\gamma$ level. ST21 infection stimulated the production of ST21 specific IgA and $\operatorname{IgM}$ in the serum (Fig. 5; $\mathrm{P}<0.05$ ) whilst all spice aqueous extract treatments significantly decreased serum $\operatorname{IgA}$ and $\operatorname{IgM}$ levels (Fig. 5; $\mathrm{P}<0.05$ ).
Spice aqueous extracts reduce the invasion ability of ST21 and decrease TNF- $\alpha$ levels of infected macrophages. In order to further elucidate the possible mechanisms of LL, LC, MP and CC aqueous extracts against ST21, a RAW 264.7 cell infection model was established. ST21 was able to infect RAW 264.7 cells. In the co-incubation group, all of the spice aqueous extract treatment reduced the number of ST21 that successfully invaded RAW 264.7 cells (Fig. 6A). To distinguish whether the extracts affected the RAW 264.7 cells or ST21, prior to performing the invasion assay, the cells and bacteria were pretreated with spice aqueous extracts for $1 \mathrm{~h}$. The infection process was then performed without aqueous extracts co-incubation. In the cell pretreatment group, the number of invading ST21 only significantly decreased in the $\mathrm{CC}$ group. However, in the ST21-pretreatment group, the invasion abilities of ST21 were significantly decreased for all aqueous extract groups (Fig. 6A). In order to clarify the effects of four spices in regulating TNF- $\alpha$ levels for ST21 eradication, specific antibodies (TNF- $\alpha$ depletion group) were added to deplete TNF- $\alpha$ in the cell culture (Fig. 6A). Compared with the co-incubation group, the invasion abilities of ST21 increased in LC and infection groups, when 
A

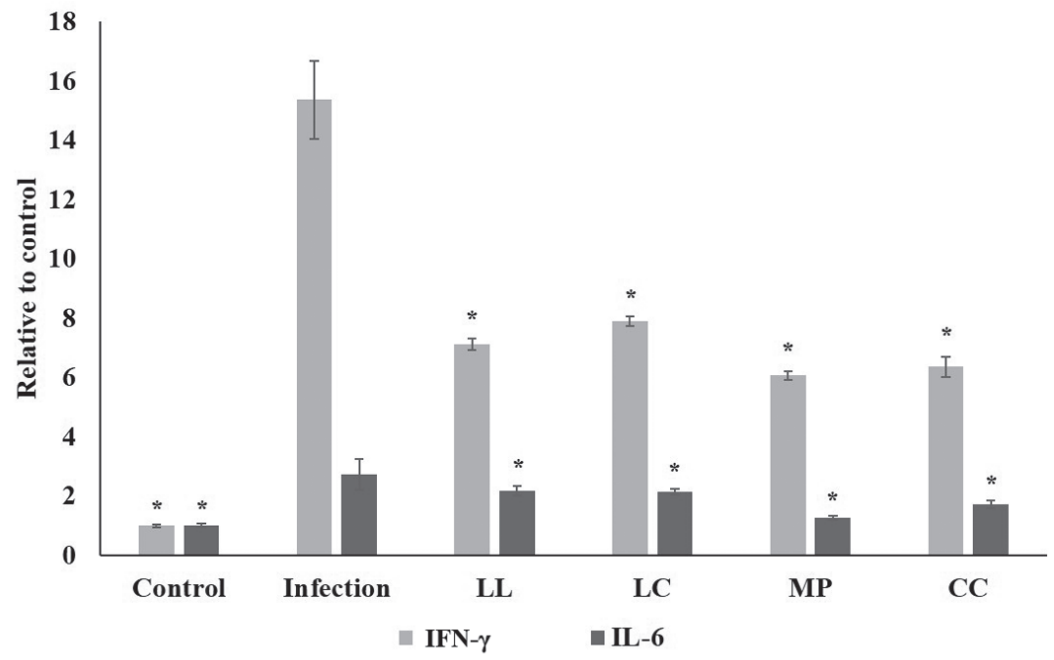

B

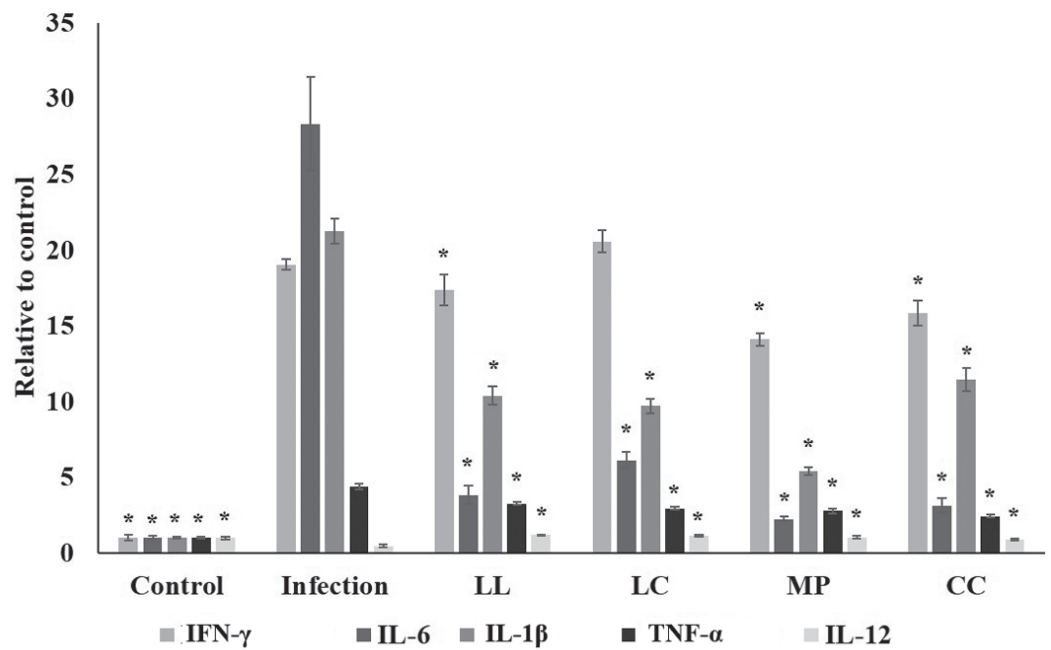

Figure 4. Spice aqueous extract treatment attenuates inflammation. (A) Serum levels of IFN- $\gamma$ and IL-6 levels, and (B) IFN- $\gamma$, IL-6, IL-1 $\beta$, TNF- $\alpha$ and IL-12 levels in the spleen of mice without infection (control), ST21-infected mice without any treatment (infection) or following LL, LC, MP or CC aqueous extract treatment $(\mathrm{n}=10) .{ }^{*} \mathrm{P}<0.05$ vs. the infection group. IFN, interferon; IL, interleukin; TNF, tumor necrosis factor; ST21, Salmonella enterica serovar Typhimurium strain ST21; LL, Ligustrum lucidum Ait; LC, Lysimachia christinae Hance; MP, Mentha piperita Linn; CC, Cinnamomum cassia Presl.

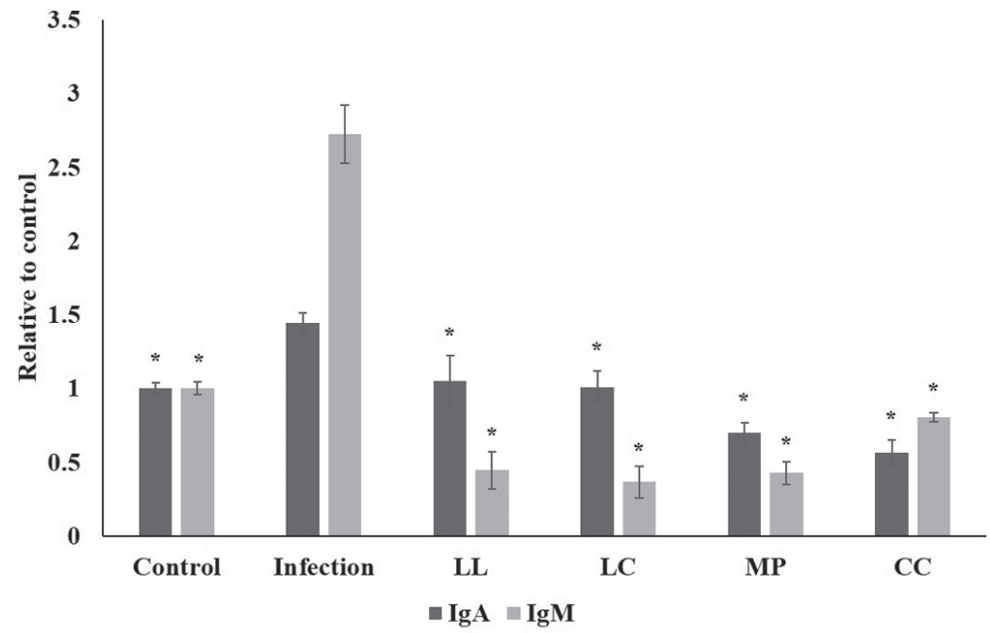

Figure 5. Spice aqueous extract treatment decreases Ig serum levels. Serum levels of IgA and IgM in mice without infection (control), ST21-infected mice without any treatment (infection) or following LL, LC, MP or CC aqueous extract treatment ( $\mathrm{n}=10)$. ${ }^{*} \mathrm{P}<0.05$ vs. the infection group. Ig, immunoglobulin; ST21, Salmonella enterica serovar Typhimurium strain ST21; LL, Ligustrum lucidum Ait; LC, Lysimachia christinae Hance; MP, Mentha piperita Linn; CC, Cinnamomum cassia Presl. 


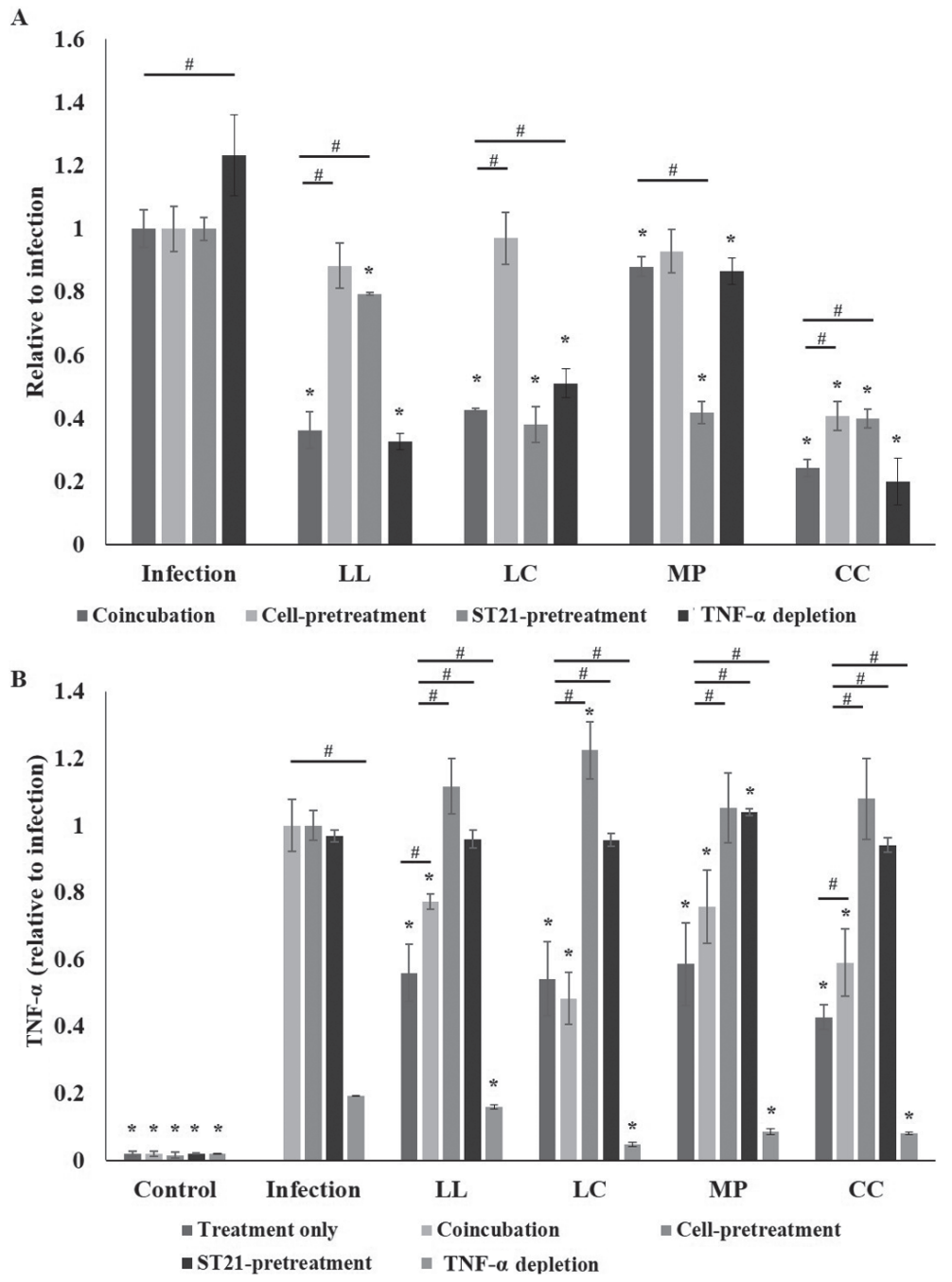

Figure 6. Spice aqueous extracts reduce the invasion ability of ST21 and decrease TNF- $\alpha$ levels of infected macrophages in vitro. (A) ST21 invasion ability and (B) TNF- $\alpha$ expression levels of ST21-infected RAW264.7 cells without any treatment (infection), and treated with LL, LC, MP or CC aqueous extract for $1 \mathrm{~h}$. Coincubation groups refer to the cells treated with spice aqueous extracts alongside ST21 infection. Cell-pretreatment groups were pretreated by spice aqueous extracts prior to ST21 infection. ST21-pretreatment groups were pretreated with spice aqueous extracts prior to infecting cells. Treatment only groups are the cells treated with spice aqueous extracts only $(n=3) .{ }^{*} \mathrm{P}<0.05$ vs. the infection group. ${ }^{*}$ Means significantly different from the coincubation group with the same treatment, $\mathrm{P}<0.05$. ST21, Salmonella enterica serovar Typhimurium strain ST21; TNF, tumor necrosis factor; LL, Ligustrum lucidum Ait; LC, Lysimachia christinae Hance; MP, Mentha piperita Linn; CC, Cinnamomum cassia Presl.

the level of TNF- $\alpha$ was depleted (Fig. 6A). All of the spice aqueous extract only treatments reduced TNF- $\alpha$ levels of RAW 264.7 cells (Fig. 6B). Compared with the co-incubation group, TNF- $\alpha$ levels increased in both cell- and ST21-pretreatment groups (Fig. 6B).

\section{Discussion}

The present study demonstrated that LL, LC, MP and CC aqueous extracts displayed weak in vitro inhibitory and bactericidal activity against ST21, as evidenced by the high MIC and $\mathrm{MBC}$ values. However, the in vivo study determined that 7 days of pretreatment and 4 days post-treatment with spice aqueous extracts significantly improved body weight loss, decreased ST21 count in feces, blood and organs as well as decreasing the production of inflammatory cytokines and Igs in blood. It was hypothesized that the spice aqueous extracts underwent in vivo metabolism to produce the necessary bioactive compounds, which may explain for the observed differences compared with the in vitro model. To the best of our knowledge, the present study was the first to demonstrate the anti-ST21 effects of LL, LC, MP and CC aqueous extracts.

ST21 can infect the blood circulatory system and organs, which in turn stimulates a systemic immune and inflammatory response $(1,16)$. The present study corroborated the literature where it was demonstrated that greater ST21 counts and over-production of Igs and cytokines in blood and organs were detected following infection $(18,19)$. Results suggested that pretreatment with spice aqueous extracts may result in the deposition of antibacterial compounds, that subsequently repressed the onset of ST21 infection and decreased fecal ST21 counts. LL and MP aqueous treatments had lower MIC values than the other spices and decreased ST21 counts in blood, liver and spleen. Thus, it is probable that LL and MP possessed a higher level of anti-ST compounds and thus exhibited greater efficiency in reducing ST21 counts. It was also determined that all four spice aqueous extracts contained substantial levels of phenolic acids and flavonoids. The anti-ST effect of oleanolic 
acid, a triterpenoic acid, has been previously reported (20) and may be responsible for the antioxidant and anti-inflammatory effects of LL (21). Therefore, the observed lower MIC value of LL, and greater reduction in fecal and blood ST21 counts in LL treated mice might be partially due to the action of oleanolic acid. Although MP essential oil has anti-Salmonella effects (11), the active compounds remain unknown. It is possible that the aqueous extract used in the present study contained the bioactive compound(s), which lowered MIC value and induced greater ST21 clearance in feces and blood, but this needs to be validated in future studies.

In the present study, ST21 infection triggered the release of several inflammatory cytokines including IFN- $\gamma$, IL- 6 , IL- $1 \beta$ and TNF- $\alpha$ in the blood and spleen, which revealed that infection induced systemic inflammatory stress. However, LL, LC, MP and CC aqueous extracts significantly decreased these inflammatory factor levels with only a mild effect observed in spleen IFN- $\gamma$ levels. These results strongly suggested that these aqueous extracts effectively attenuated ST21-induced inflammation in the circulatory system and organs. It has been reported that anti-inflammatory phytochemicals such as ursolic acid, oleanolic acid, quercetin, cinnamic acid, rosmarinic acid and coumarin are present in LL, LC, MP and CC (22-24). The present study identified that these spices were rich in phenolic acids and flavonoids and it is likely that an anti-inflammatory effect was exerted. The subsequent effect was a decrease in IFN- $\gamma$, IL-6, IL- $1 \beta$ and TNF- $\alpha$ levels, which alleviated ST21-induced inflammation, except LC treatment did not suppress ST21-induced IFN- $\gamma$ expression. Chang et al (25) determined that IL-12 possesses a potential anti-inflammatory function because the expression of IL-10, which limits inflammatory immune responses, was conditional on the presence of IL-12 in re-stimulated Th1 memory cells. The present study determined that LL, LC, MP and CC aqueous treatments significantly increased spleen IL-12 production in ST21 infected mice, which may have enhanced the anti-inflammatory effect.

Igs, produced by the host immune system, are involved in the response against Salmonella infection $(18,19)$. In the present study, the overproduction of $\operatorname{IgA}$ and $\operatorname{IgM}$ in the blood of ST21 infected mice indicated the activation of the immune response. LL, LC, MP and CC aqueous extract treatments repressed the formation of $\operatorname{IgA}$ and $\operatorname{IgM}$ in blood. It is possible that the spice aqueous extracts decreased ST21 counts and ameliorated the inflammatory status of infected mice, which in turn diminished the burden on the host immune system and reduced $\operatorname{IgA}$ and IgM production. Maghraby and Bahgat (26) determined that coumarin, an active component of $\mathrm{CC}$, had an immunomodulatory effect at both humoral and cellular levels in mice infected by Schistosoma mansoni. It was also reported that MP crude leaf extract improved Schistosoma mansoni infection in mice via an immunomodulatory effect (27). The spices contained phenolic acids and flavonoids therefore it is also possible that the active compound(s) exerted immune-like actions, which in turn alleviated the production of Igs in blood of ST21-infected mice. However, the total content of phenolic acids and flavonoids in blood were too low to be detected, therefore, it is difficult to conclude the roles of phenolic acids and flavonoids in this study.

The possible mechanisms of LL, LC, MP, and CC against ST21 were investigated via an in vitro model. CC aqueous extract moderated the virulence activity of ST21 and improved the ability of macrophages against ST21 infection. CC treatment suppressed ST21 invasion into intestinal tissues more significantly than the other spice treatments. MP aqueous extract exhibited both bactericidal activity and the ability to suppress ST21 invasion, which resulted in a reduction of ST21 load in mouse feces and organs. LL aqueous extract demonstrated a similar mechanism against ST21 infection but with a weaker effect in attenuating the virulence of ST21 compared with MP aqueous extract. LC aqueous extract only moderately decreased ST21 invasion, therefore, the treatment did not inhibit ST21 infection as efficiently as other treatments in vivo. In the early phase of infection, the expression of TNF- $\alpha$ in macrophages facilitates the eradication of intracellular Salmonella (5). The number of intracellular ST21 was increased compared with the coincubation group following LC aqueous extract treatment and TNF- $\alpha$ depletion therefore, the protective effect of LC against ST21 infection may involve TNF- $\alpha$ regulation. Furthermore, all four spice aqueous extracts induced TNF- $\alpha$ expression in macrophages, which indicated that they might trigger the activation of macrophages through TNF- $\alpha$ expression regulation. Although the bactericidal activities of LL, LC, MP, and CC aqueous extracts were weak, they were still able to alleviate ST21 by modulating the activation of macrophages. The mechanisms and potential compounds involved in this infection attenuation require further investigation.

Boiled LL, LC, MP, and CC are commonly used in food preparation and folk medicine in many Asian countries. Therefore, investigation into the protective effects of the aqueous extracts is more relevant and also cheaper than isolated pure active compounds. Although the application of these aqueous extracts is feasible and may prove useful to prevent and attenuate Salmonella infection, the present study did not monitor the impact of these aqueous extracts on mouse liver and kidney functions. For example, the hepatic toxicity of coumarin, an active compound of $\mathrm{CC}$ has been reported previously (28), therefore, further in vivo studies are necessary to further examine the metabolism, safety and efficiency of these aqueous extracts before they can be widely used against Salmonella infection.

In conclusion, LL, LC, MP, and CC aqueous extract treatment attenuated ST21 infection in mice via decreasing bacterial counts, reducing inflammatory stress and lowering Ig production. LL and MP aqueous extract treatments demonstrated the most significant effect on eradicating ST21 infection. All four aqueous extracts demonstrated different mechanisms in attenuating ST21 invasion. The present study, to the best of our knowledge, is the first to demonstrate the protective effects of LL, LC, MP, and CC aqueous extracts in alleviating a common food-borne disease. All four spices are widely available and the aqueous extracts easily prepared. The present findings suggested that LL, LC, MP and CC may be considered as potent functional foods with anti-Salmonella effects, but this needs to be investigated further.

\section{Acknowledgements}

The authors would like to acknowledge Dr. Yit Lung Khung (Department of Biological Science and Technology, China 
Medical University, Taiwan) for valuable suggestions on this work and helping the authors with the English language editing of the manuscript.

\section{Funding}

The present study was supported by China Medical University Hospital (grant no. DMR-106-127) and the Tainan Municipal Hospital (grant no. 105-10).

\section{Availability of data and materials}

The datasets generated and/or analyzed during the current study are available from the corresponding author on reasonable request.

\section{Authors' contributions}

YMH and CHC designed this study, JHF and CHS performed experiments. MCY analyzed and interpreted the data, and wrote the manuscript.All authors read and approved the final manuscript.

\section{Ethics approval and consent to participate}

The study was approved by the China Medical University animal care and use committee (permission no. 105-23).

\section{Patient consent for publication}

Not applicable.

\section{Competing interests}

The authors declare that they have no competing interests.

\section{References}

1. Heithoff DM, Shimp WR, Lau PW, Badie G, Enioutina EY, Daynes RA, Byrne BA, House JK and Mahan MJ: Human Salmonella clinical isolates distinct from those of animal origin. Appl Environ Microbiol 74: 1757-1766, 2008.

2. LaRock DL, Chaudhary A and Miller SI: Salmonellae interactions with host processes. Nat Rev Microbiol 13: 191-205, 2015.

3. Keestra-Gounder AM, Tsolis RM and Baumler AJ: Now you see me, now you don't: The interaction of Salmonella with innate immune receptors. Nat Rev Microbiol 13: 206-216, 2015.

4. Beal RK, Wigley P, Powers C, Barrow PA and Smith AL: Cross-reactive cellular and humoral immune responses to Salmonella enterica serovars Typhimurium and Enteritidis are associated with protection to heterologous re-challenge. Vet Immunol Immunopathol 114: 84-93, 2006.

5. Lalmanach AC and Lantier F: Host cytokine response and resistance to Salmonella infection. Microbes Infect 1: 719-726, 1999.

6. Krzyzanowska J, Janda B, Pecio L, Stochmal A, Oleszek W, Czubacka A, Przybys M and Doroszewska T: Determination of polyphenols in Mentha longifolia and M. piperita field-grown and in vitro plant samples using UPLC-TQ-MS. J AOAC Int 94: 43-50, 2011.

7. Xia EQ, Yu YY, Xu XR, Deng GF, Guo YJ and Li HB: Ultrasound-assisted extraction of oleanolic acid and ursolic acid from Ligustrum lucidum Ait. Ultrason Sonochem 19: 772-776, 2012.

8. Jurikova T, Mlcek J, Skrovankova S, Balla S, Sochor J, Baron M and Sumczynski D: Black crowberry (Empetrum nigrum L.) flavonoids and their health promoting activity. Molecules 21: E1685, 2016.
9. An HJ, Jeong HJ, Um JY, Park YJ, Park RK, Kim EC, Na HJ, Shin TY, Kim HM and Hong SH: Fructus Ligustrum lucidi inhibits inflammatory mediator release through inhibition of nuclear factor-kappaB in mouse peritoneal macrophages. J Pharm Pharmacol 59: 1279-1285, 2007.

10. Kozłowska M, Laudy AE, Przybył J, Ziarno M and Majewska E: Chemical composition and antibacterial activity of some medicinal plants from lamiaceae family. Acta Pol Pharm 72: 757-767, 2015.

11. Akdemir Evrendilek G: Empirical prediction and validation of antibacterial inhibitory effects of various plant essential oils on common pathogenic bacteria. Int J Food Microbiol 202: 35-41, 2015.

12. Alzoreky NS and Nakahara K: Antibacterial activity of extracts from some edible plants commonly consumed in Asia. Int J Food Microbiol 80: 223-230, 2003.

13. Chang $\mathrm{CH}, \mathrm{Yu} \mathrm{B}, \mathrm{Su} \mathrm{CH}$, Chen DS, Hou YC, Chen YS and Hsu YM: Coptidis rhizome and Si Jun Zi Tang can prevent Salmonella enterica serovar Typhimurium infection in mice. PLoS One 9: e105362, 2014

14. Sreelatha $S$ and Padma PR: Antioxidant activity and total phenolic content of Moringa oleifera leaves in two stages of maturity. Plant Foods Hum Nutr 64: 303-311, 2009.

15. Yin MC, Chang CH, Su CH, Yu B and Hsu YM: Pteris multifida, Cortex phellodendri, and probiotics attenuated inflammatory status and immunity in mice with a Salmonella enterica serovar Typhimurium infection. Biosci Biotechnol Biochem: 1-12, 2018.

16. Doyle MP and Erickson MC: Reducing the carriage of foodborne pathogens in livestock and poultry. Poult Sci 85: 960-973, 2006

17. Kuda T, Kosaka M, Hirano S, Kawahara M, Sato M, Kaneshima T, Nishizawa M, Takahashi H and Kimura B: Effect of sodium-alginate and laminaran on Salmonella Typhimurium infection in human enterocyte-like HT-29-Luc cells and BALB/c mice. Carbohydr Polym 125: 113-119, 2015.

18. Drago-Serrano ME, Rivera-Aguilar V, Reséndiz-Albor AA and Campos-Rodríguez R: Lactoferrin increases both resistance to Salmonella typhimurium infection and the production of antibodies in mice. Immunol Lett 134: 35-46, 2010.

19. Wijburg OL, Uren TK, Simpfendorfer K, Johansen FE, Brandtzaeg P and Strugnell RA: Innate secretory antibodies protect against natural Salmonella typhimurium infection. J Exp Med 203: 21-26, 2006.

20. Senthilkumar PK and Reetha D: Isolation and identification of antibacterial compound from the leaves of Cassia auriculata. Eur Rev Med Pharmacol Sci 15: 1034-1038, 2011.

21. Senthil S, Sridevi M and Pugalendi KV: Cardioprotective effect of oleanolic acid on isoproterenol-induced myocardial ischemia in rats. Toxicol Pathol 35: 418-423, 2007.

22. Dorman HJ, Kosar M, Baser KH and Hiltunen R: Phenolic profile and antioxidant evaluation of Mentha x piperita L. (peppermint) extracts. Nat Prod Commun 4: 535-542, 2009.

23. Sandhiutami NM, Moordiani M, Laksmitawati DR, Fauziah N, Maesaroh M and Widowati W: In vitro assesment of anti-inflammatory activities of coumarin and Indonesian cassia extract in RAW264.7 murine macrophage cell line. Iran J Basic Med Sci 20: 99-106, 2017.

24. Wang J, Zhang Y, Zhang Y, Cui Y, Liu J and Zhang B: Protective effect of Lysimachia christinae against acute alcohol-induced liver injury in mice. Biosci Trends 6: 89-97, 2012.

25. Chang HD, Helbig C, Tykocinski L, Kreher S, Koeck J, Niesner U and Radbruch A: Expression of IL-10 in Th memory lymphocytes is conditional on IL-12 or IL-4, unless the IL-10 gene is imprinted by GATA-3. Eur J Immunol 37: 807-817, 2007.

26. Maghraby A and Bahgat M: Immunostimulatory effect of coumarin derivatives before and after infection of mice with the parasite Schistosoma mansoni. Arzneimittelforschung 54: 545-550, 2004

27. Dejani NN, Souza LC, Oliveira SR, Neris DM, Rodolpho JM, Correia RO, Rodrigues V, Sacramento LV, Faccioli LH, Afonso A and Anibal FF: Immunological and parasitological parameters in Schistosoma mansoni-infected mice treated with crude extract from the leaves of Mentha x piperita L. Immunobiology 219: 627-632, 2014

28. Lake BG, Evans JG, Chapuis F, Walters DG and Price RJ: Studies on the disposition, metabolism and hepatotoxicity of coumarin in the rat and Syrian hamster. Food Chem Toxicol 40: 809-823, 2002.

This work is licensed under a Creative Commons Attribution-NonCommercial-NoDerivatives 4.0 International (CC BY-NC-ND 4.0) License. 